\title{
INTERPRETATION OF CARBON STARS SPECTRA FROM MODEL ATMOSPHERES COMPUTATIONS*
}

\author{
F. QUERCI and M. QUERCI \\ Laboratoire du Télescope Infrarouge, D.E.P.E.G., Observatoire de Paris, Meudon, France
}

\section{Introduction}

The first part of this paper presents a grid of model atmospheres for carbon stars in which the most striking feature is the inclusion of the molecular line blanketing effect of $\mathrm{CO}, \mathrm{CN}$ and $\mathrm{C}_{2}$ through opacity probability distribution functions. The techniques and the main results have been fully discussed in previous papers (Querci, 1972; Querci et al., 1972 and 1973), and consequently we will give just a rapid description asking the reader to refer to these papers for details. However, the grid has been extended here to lower temperatures.

The second part presents original results from the M. Querci's 'Thèse d'Etat' (1974), and concerns the computation of synthetic spectra. A kind of grid of synthetic spectra has been computed from each model atmosphere, that is to say for each particular effective temperature and gravity, over a selected spectral range. The influence of the gravity and of the effective temperature on the computed spectra and the problem of the line cutting procedure are considered. Finally, comparisons with a high resolution infrared spectrum of a carbon star are made.

The conclusion summarizes the experimental progress that must be made in order to be able to make meaningful comparisons between predictions and observations.

\section{The Grid of Model Atmospheres}

In the construction of the model atmospheres, in order to take into account the blanketing by about $10^{6}$ lines of the three following diatomic molecules,

$\mathrm{CN}$ (red and violet systems),

$\mathrm{CO}$ (fundamental, first and second overtones),

$\mathrm{C}_{2}$ (Swan, Phillips and Ballik-Ramsay systems),

for all the isotopic species observed in carbon stars, we have adapted the statistical method proposed by Ström and Kurucz (1966).

As explained in Querci et al. $(1972,1974)$ and Querci $(1972)$, for each subinterval of the primary spectral intervals dividing the total spectral range, we build up and put on a magnetic disk a table of opacity probability distribution functions consisting of a grid in temperature $T$ and gas pressure $P_{g}$, in which the values required during the run of the model are obtained by interpolation.

The lines used for building the OPDF tables are put on a magnetic tape, the ATLAS

* Presented by F.Q. 
tape. The selection of the lines is such that, for a given pair of values of temperature and gas pressure, $T_{1}-P_{g_{1}}$, which furnish a maximum number of lines for a given molecular system, we keep those lines for which: $l_{v_{0}} \geqslant 0.1 k_{v_{\min }}\left(T_{1}, P_{g_{1}}\right)$, where $l_{v_{0}}$ is the monochromatic absorption coefficient at the line center and $k_{v_{\min }}$ is the minimum continuous absorption coefficient over the spectral range from 0.2 to $20 \mu$. Of the lines previously stored on the ATLAS tape, within each primary interval, $\Delta v$, we keep the lines such that $l_{v_{0}} \geqslant 0.1 k_{v}\left(T, P_{g}\right)$. The line wings are also cut when $l_{v}<0.1 k_{v}$, where $k_{v}$ is the continuous absorption coefficient per gramme of stellar material in $\Delta v$.

Sixty primary intervals $\Delta v$ divide the total spectral range and the molecular absorption is smoothed within each interval. The subintervals of $\Delta v$ have equal statistical weights.

The OPDF tables have been established for:

$$
T=840,1006,1260,1680,2100,2800,4200,6300,8400 \mathrm{~K},
$$

and

$$
\log P_{g}=-3,-1,+1,+3,+5 .
$$

The abundances have been taken to be:

$$
\begin{aligned}
& \mathrm{C} / \mathrm{H}=4.1 \times 10^{-5}, \quad \mathrm{~N} / \mathrm{C}=37.0, \quad \mathrm{C} / \mathrm{O}=3.2, \\
& \mathrm{C}^{12} / \mathrm{C}^{13}=1.11 \times 10, \quad \mathrm{~N}^{15} / \mathrm{N}^{14}=4.1 \times 10^{-4}, \quad \mathrm{O}^{16} / \mathrm{O}^{18}=2.0 \times 10^{-3} .
\end{aligned}
$$

In solving the ionisation and dissociation equilibria, 26 atoms (neutral and once ionized) and 200 molecules, respectively, have been included (from Tsuji, 1973).

The microturbulence velocity has a single value of $5 \mathrm{~km} \mathrm{~s}^{-1}$.

The model atmospheres presented are characterized by the effective temperatures:

$$
T_{e}=4500,4200,3800,3400,3000,2600,2200 \mathrm{~K},
$$

and the gravities:

$$
g=0.1,1.0,10.0 \text {. }
$$

The lower temperature model atmospheres have to be considered as preliminary to more realistic models in which polyatomic opacities are included.

The first value of the standard optical depth has been taken to be $\log \tau_{\mathrm{std}}=-6.0$ and the standard wavelength to be $0.8 \mu$.

The models are calculated with 80 optical depth points.

The chemical composition is the same as in the computation of the table of opacity distribution functions.

The total pressure used in this work consists of gas, radiation and turbulent pressures. The equation of hydrostatic equilibrium is integrated by Hamming's method.

The radiative flux $F$ through a layer is computed by summation of the fluxes $F_{v}$ corresponding to each spectral interval $\Delta v$ :

$$
F_{v}=\sum_{i=1}^{n} W_{i} F_{v}^{i}
$$


where $F_{v}^{i}$ are the elementary fluxes, $W_{i}$ are the weights and $n$ is the number of subintervals in $\Delta v$.

The elementary fluxes imply the source function, that is calculated by the Feautrier difference equation method.

I have selected some of the results for illustration of the grid. Others are discussed in Querci (1972) and Querci et al. (1974).

Figure 1 shows the $T(\tau)$ laws of the grid of model atmospheres. Figures 2 and 3 present the run of the physical quantities with the standard optical depth for effective temperatures corresponding to very cool atmospheres: $T_{e}=2600 \mathrm{~K}$ and $T_{e}=2200 \mathrm{~K}$ with $g=1.0$ and 10 . The run of the $T(\tau)$ laws and of the logarithm of the density $\varrho$ are also presented. The variation of the temperatures is well correlated with that of the partial pressures, and we see how large variation in the absorption in the molecular lines can modify the blanketing effect.

To illustrate the importance of blanketing in the coolest stars, model atmospheres for $T_{e}=4500,3400$ and $2600 \mathrm{~K}$ and $g=1$ have been run with and without line blanketing by $\mathrm{CO}, \mathrm{CN}$ and $\mathrm{C}_{2}$. The curves in Figure 4 also show the importance of surface cooling and deep backwarming. In the deepest layers of the star, where the temperature is so high that no lines can be formed, the unblanketed and the blanketed models are obviously very similar.

Figure 5 presents the predicted emergent fluxes for 3 typical effective temperatures, 4500,3400 and $2600 \mathrm{~K}$, for the same gravity, $g=10$.

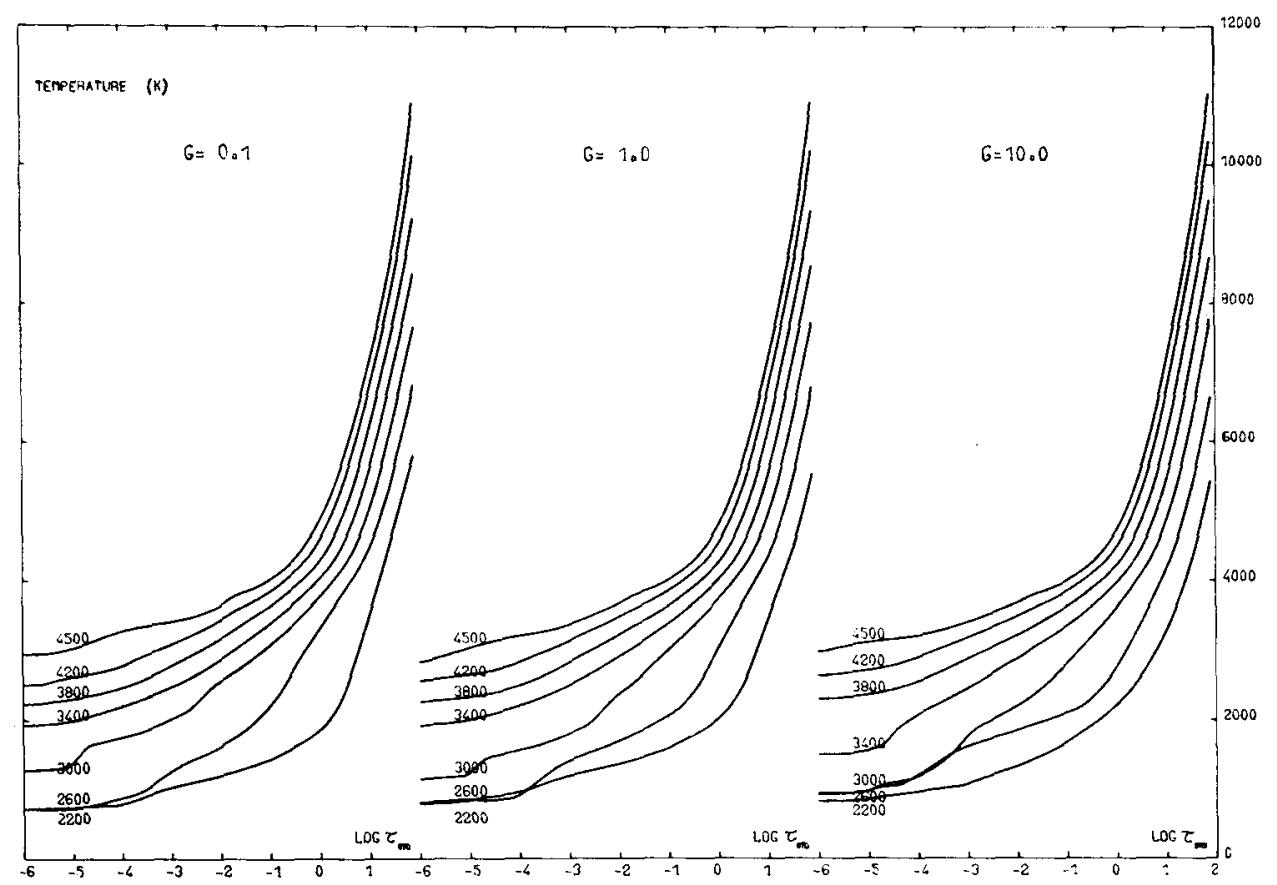

Fig. 1. $T(\tau)$ laws of the grid of model atmospheres. 


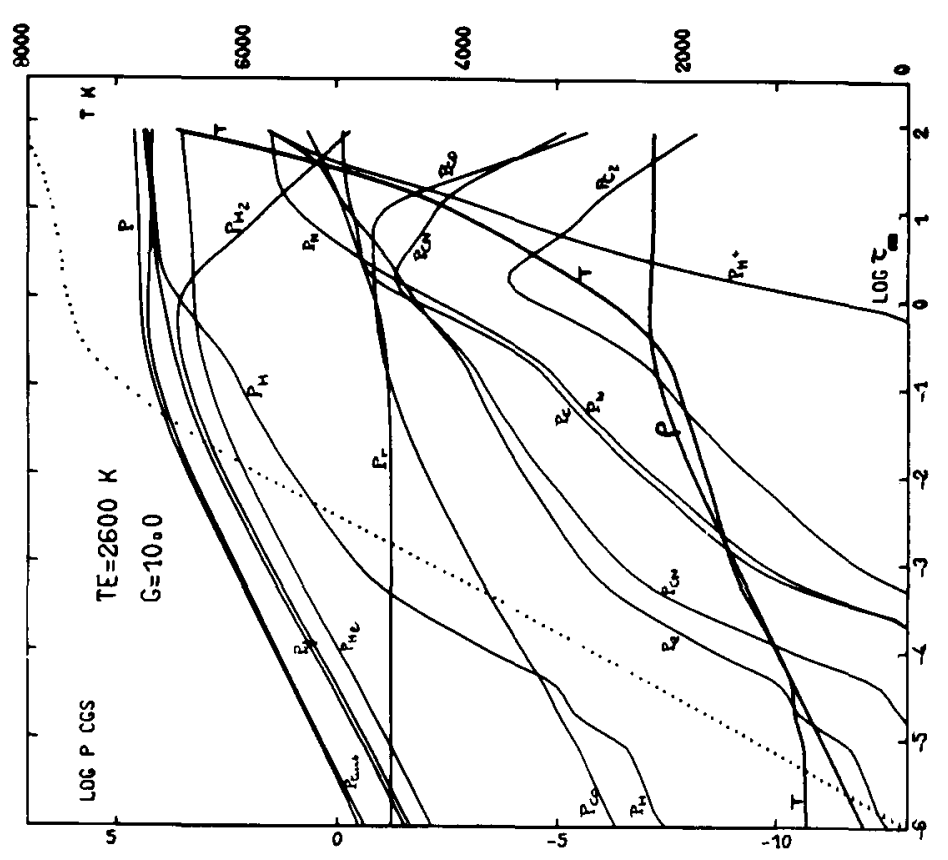

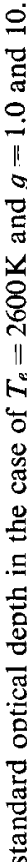

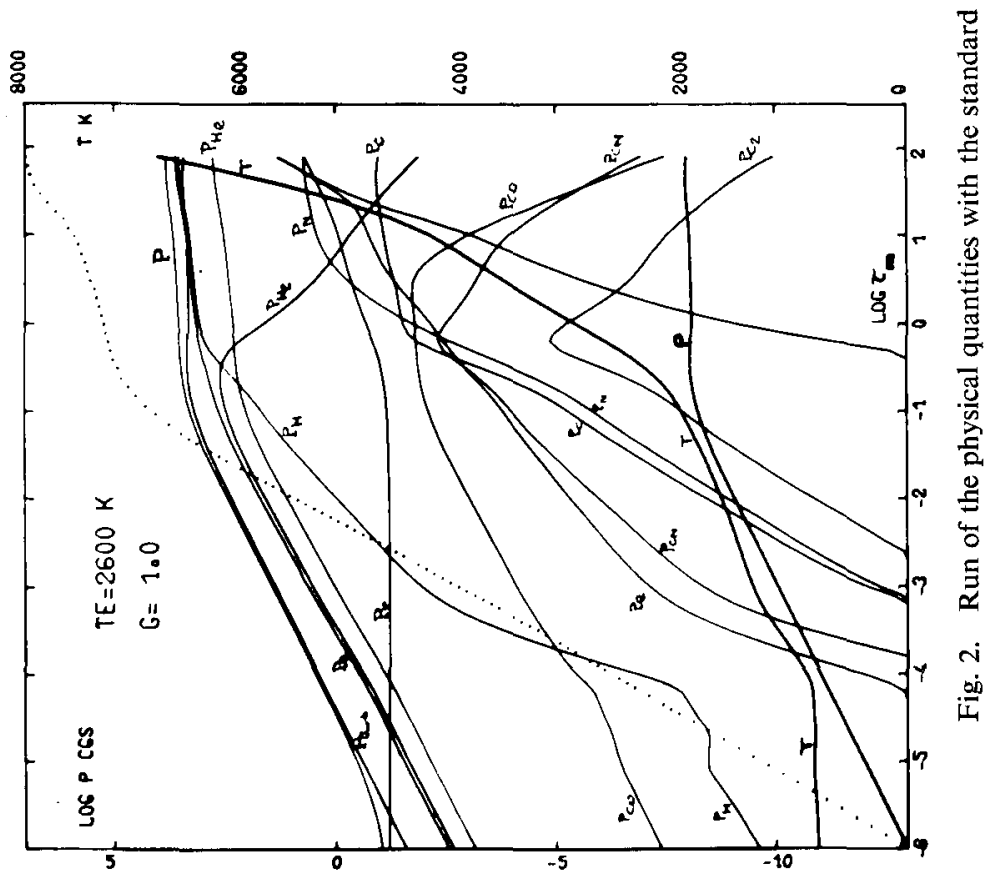




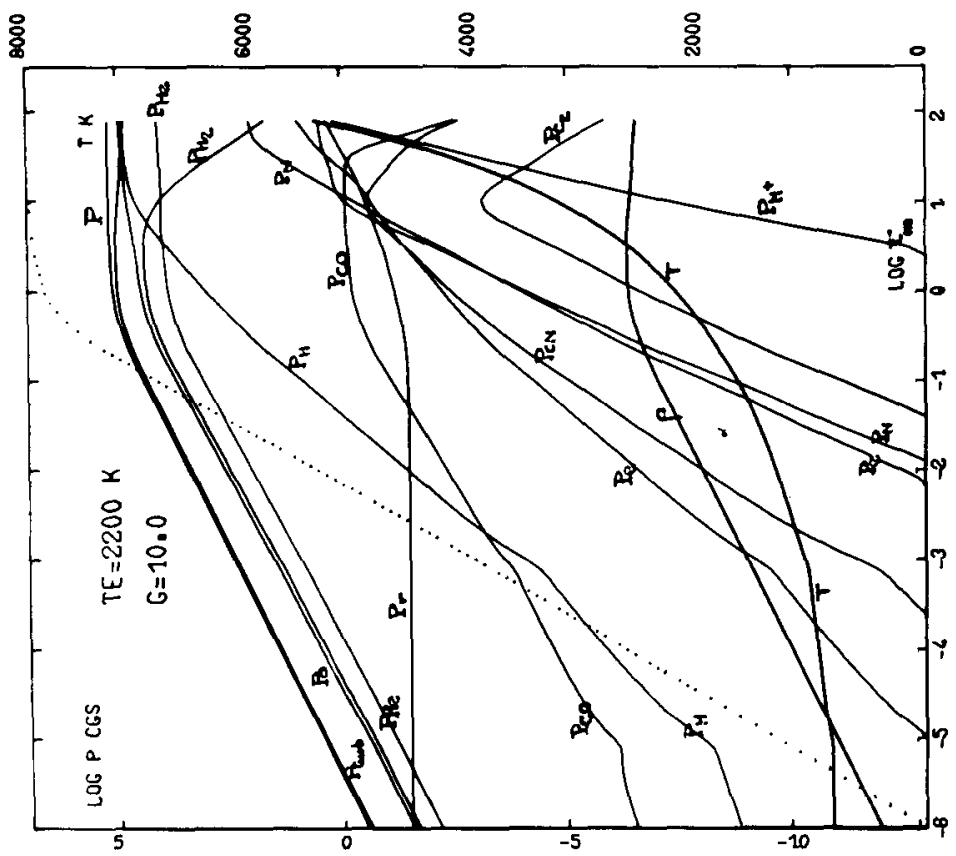

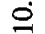
ए]

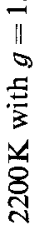



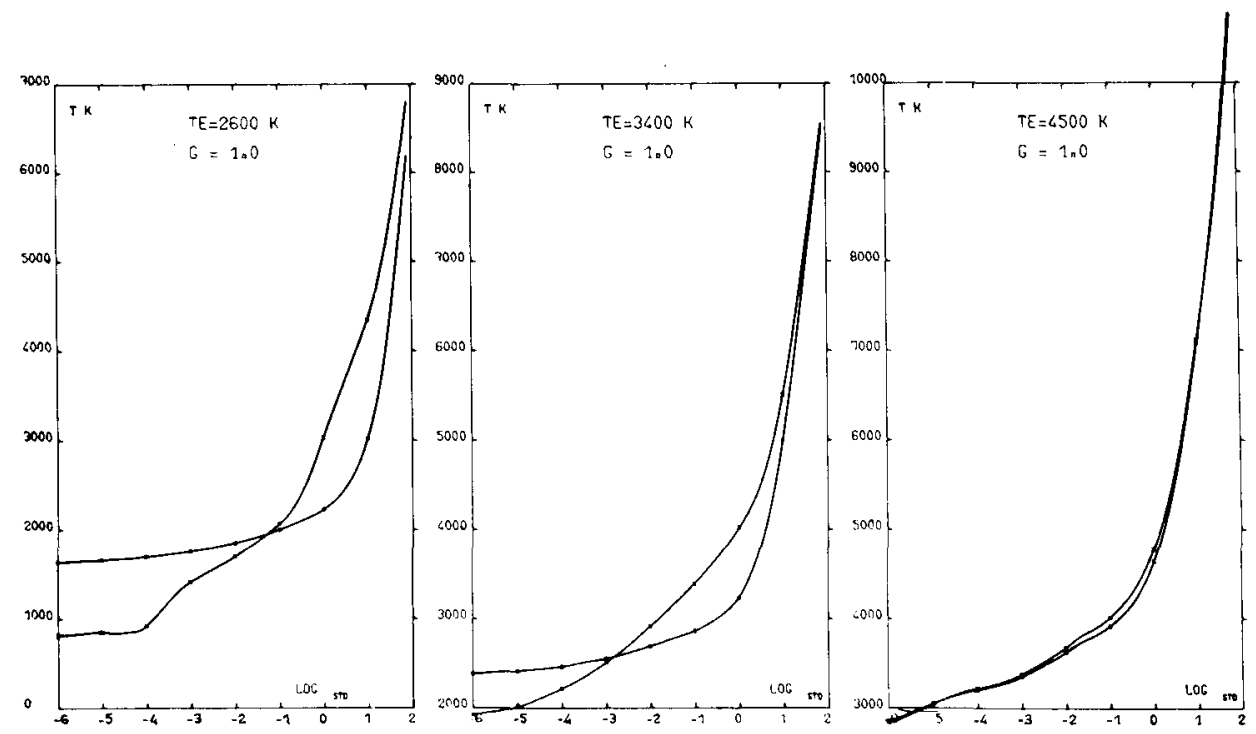

Fig. 4. $T(\tau)$ laws from the models with $T_{e}=4500 \mathrm{~K}, 3400 \mathrm{~K}$ and $2600 \mathrm{~K}$, and $g=1$, including and without including the molecular line blanketing.

While for the atmosphere with $T_{e}=4500 \mathrm{~K}$ the emitted flux stays near the blackbody curve with only the most important bands of $\mathrm{CN}$ and $\mathrm{CO}$ appearing, for $\mathrm{Te}$ $=3400 \mathrm{~K}$ the flux at $1.8 \mu$ to the right of the $\mathrm{H}^{-}$minimum absorption is heavily absorbed by $\mathrm{CN}$ and $\mathrm{CO}$. In the $2600 \mathrm{~K}$ atmosphere, the flux emitted at $1.8 \mu$ is enhanced and the molecular features appear clearly:

- the violet $\mathrm{CN}$ at $0.39 \mu$ ( 0 sequence), $0.42 \mu$ ( -1 sequence), $0.46 \mu$ ( -2 sequence),

- the sequences 5 to -4 of the red $\mathrm{CN}$,

- the $\mathrm{C}_{2}$ Swan system at $0.47 \mu, 0.51 \mu$ and $0.62 \mu$,

- the sequences $0,-1,-2$ of the $\mathrm{C}_{2}$ Ballik-Ramsay system,

- the fundamental, the first and second overtones of $\mathrm{CO}$.

By following the variation with the effective temperature, it is easy to see the influence of the molecular line blanketing by the 3 molecules $\mathrm{CO}, \mathrm{CN}$ and $\mathrm{C}_{2}$ on the fluxes emitted by carbon stars.

\section{The Synthetic Spectra}

At each optical depth point in the atmosphere, the program uses previously computed values of $\tau_{\mathrm{std}}, T(\tau), \log P_{g}$, and the standard total continuous absorption coefficients $\left(k_{v}+\sigma_{v}\right)_{s t d}$. It also uses the assumed $\mathrm{H} / \mathrm{C} / \mathrm{N} / \mathrm{O}$ abundance ratios and the isotopic abundance ratios. The number of lines considered is the number used to compute the opacity distribution functions. These lines are picked up from the ATLAS tape over the spectral region for which synthetic spectra are being computed.

For each $\tau_{\text {std }}$, various quantities are computed: the abundance of $\mathrm{CO}, \mathrm{CN}$ and $\mathrm{C}_{2}$, the continuous absorptions, the scattering and the induced absorptions. The program 


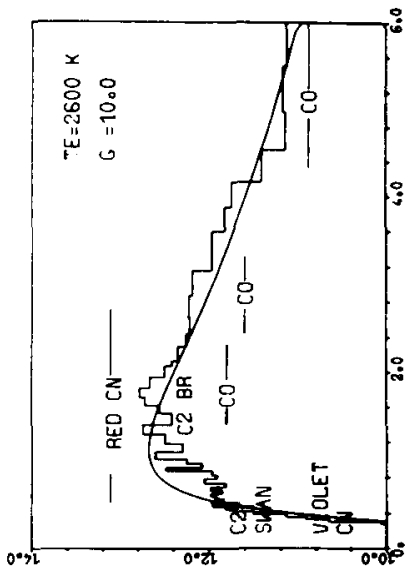

11
0
0
0
0
0
0
0
0
0
11
0
0

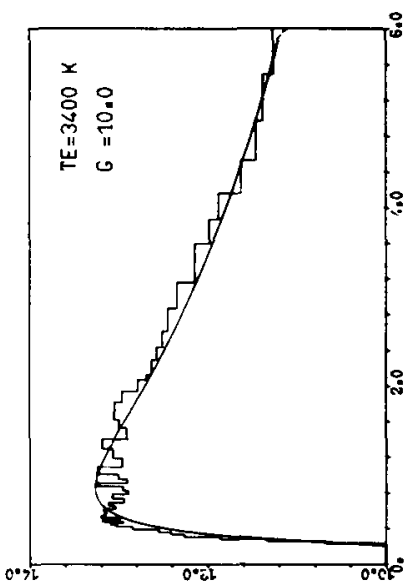

安

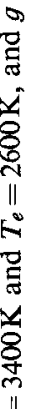

r

幽

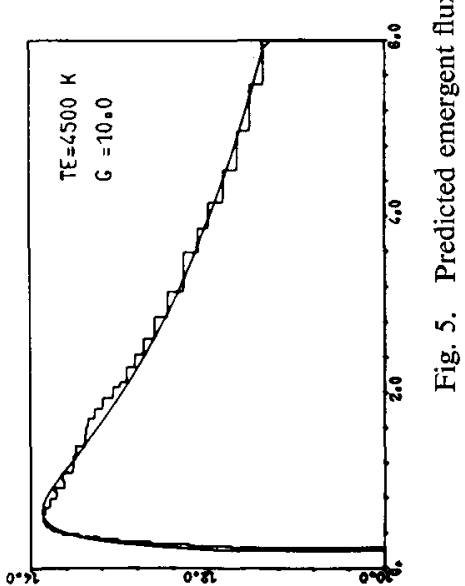


checks the width of the biggest line of the spectrum; this quantity is used to compute the contribution of all the line profiles to the line absorption coefficient $\sum l_{v}^{i}$ at the frequency $v$.

The frequency step length is set equal to the distance between two primary points of the observed spectrum $\left(\simeq 0.08 \mathrm{~cm}^{-1}\right)$.

The transfer equation at each frequency point is solved by the Feautrier difference equation method with the second order upper boundary condition from Auer (1967).

The emerging continuous flux $F_{c}$ is computed once for each spectral interval. The emerging flux $F_{v}$ in the line and the ratio $R_{v}=F_{v} / F_{c}$ are computed at each frequency point.

The computed spectra are finally convolved with the same instrumental and apodized function as the observed spectra, and the synthetic and observed spectra can then be directly compared.

To compute synthetic spectra, spectral intervals are chosen which contain characteristic molecular features. We present here computations for a spectral interval around the head of the $(2,0)$ band of the $\mathrm{CO}$ first overtone. In this spectral range, from 4319 to $4400 \mathrm{~cm}^{-1}$, the $\mathrm{CO}$ vibration-rotation spectrum and the electronic red

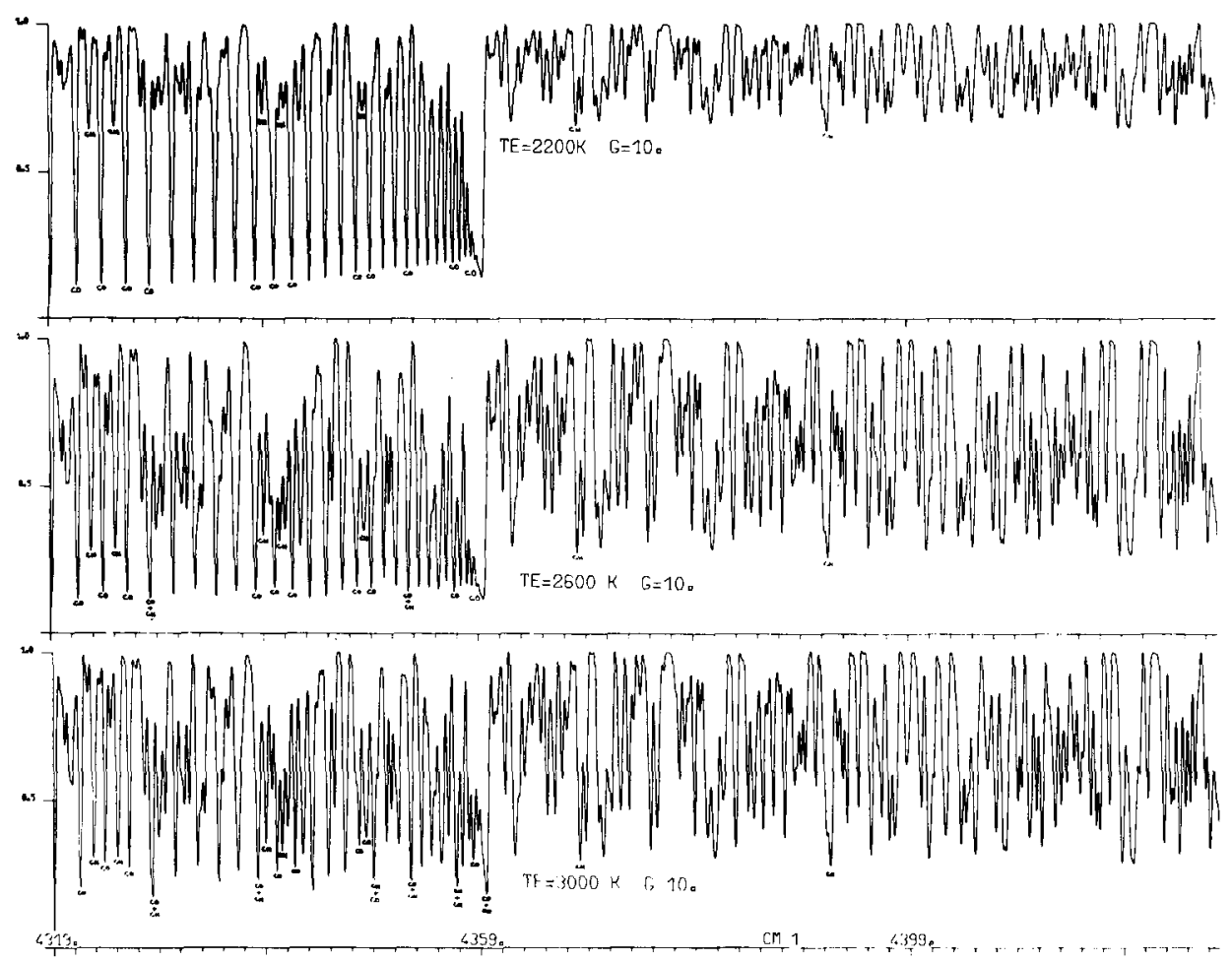

Fig. 6. Influence of the effective temperature on the synthetic spectra: $T_{e}=2200 \mathrm{~K}, 2600 \mathrm{~K}$ and $3000 \mathrm{~K}$ for $g=10$. 


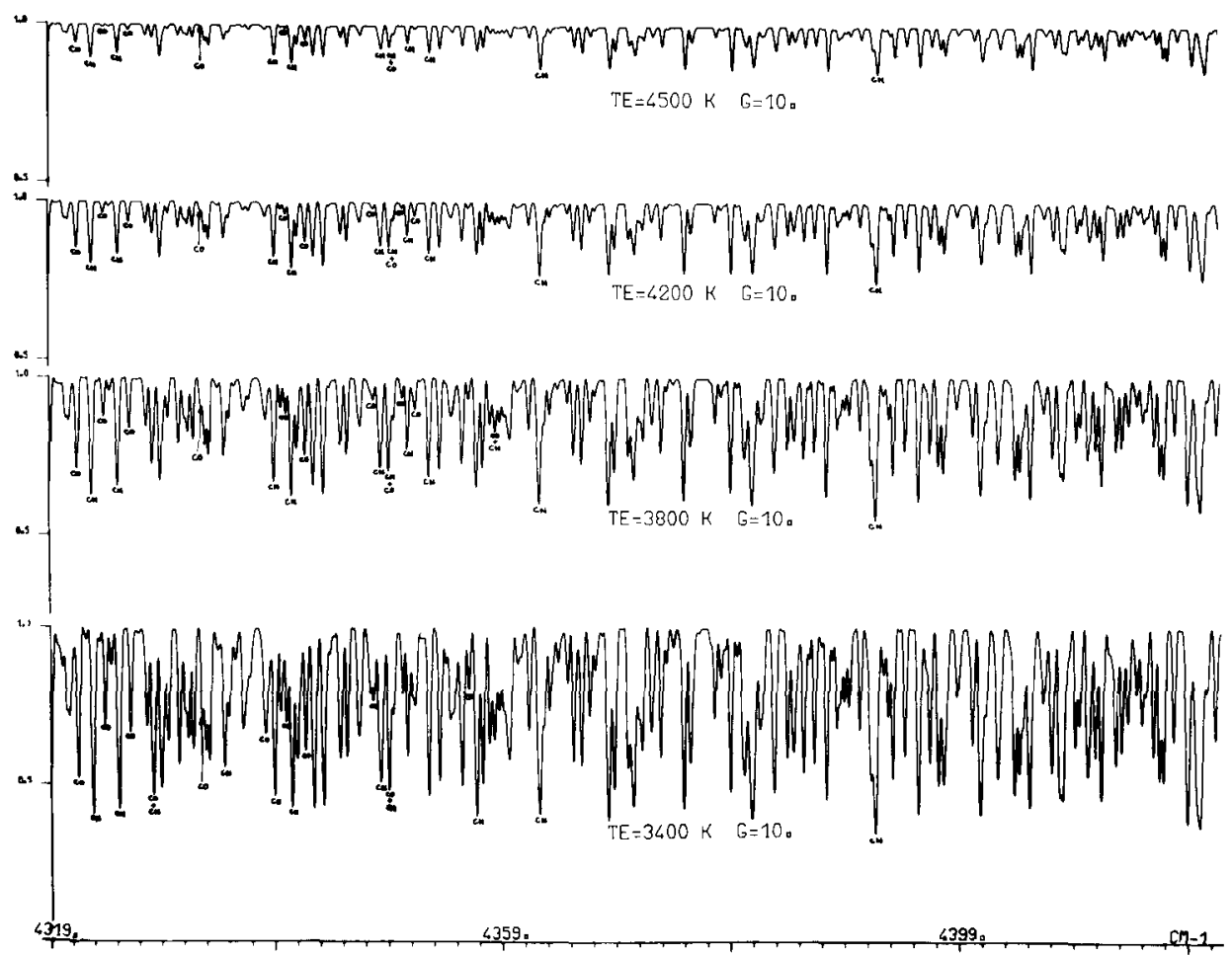

Fig. 7. Influence of the effective temperature on the synthetic spectra: $T_{e}=3400 \mathrm{~K}, 3800 \mathrm{~K}, 4200 \mathrm{~K}$ and $4500 \mathrm{~K}$ for $g=10$.

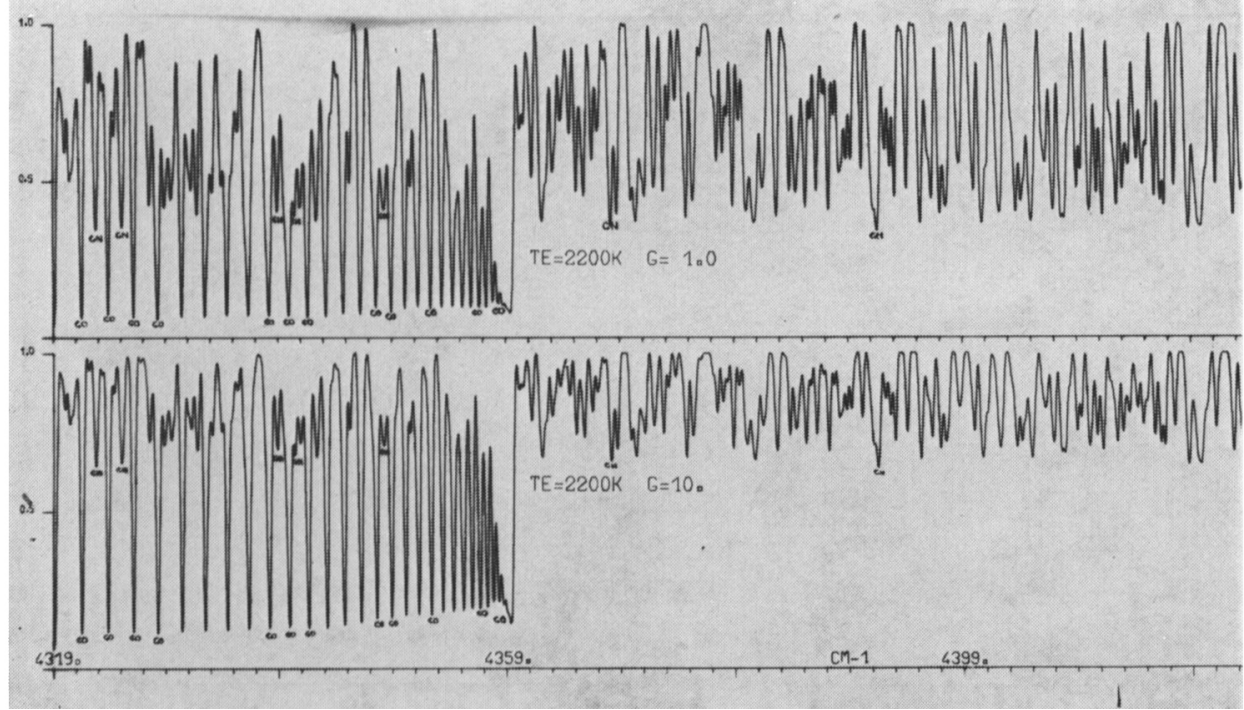

Fig. 8. Influence of the gravity on the synthetic spectra: $g=1.0$ and 10 for $T_{e}=2200 \mathrm{~K}$. 
system of $\mathrm{CN}$ are very efficient. Moreover, these sysems are the two most important absorbers in the atmospheres considered.

We note that the line wavenumbers have been corrected for the radial velocity peculiar to the molecular system considered.

First, we look at the influence of the effective temperature on the synthetic spectra.

Figure 6 shows the case of low temperatures, $T_{e}=2200,2600,3000 \mathrm{~K}$, for $g=10$. In the atmosphere with $T_{e}=2200 \mathrm{~K}$, the CO lines are very strong, while the CN lines are very weak. For $T_{e}=2600 \mathrm{~K}$, the $\mathrm{CN}$ lines become more important; the intensity of the CO lines remains the same. For $T_{e}=3000 \mathrm{~K}$, the $\mathrm{CN}$ lines and the $\mathrm{CO}$ lines have about the same strength and $\mathrm{CO}$ begins to weaken.

Figure 7 groups the following temperatures: $T_{e}=3400,3800,4200,4500 \mathrm{~K}$, for $g=10$. For $T_{e}=3400 \mathrm{~K}$, the $\mathrm{CO}$ bandhead is weaker than the $\mathrm{CN}$ lines, while for $T_{e}=4500 \mathrm{~K}$, the CO lines have nearly disappeared.

We now look at the influence of the gravity on the synthetic spectra.

It is striking that, for $T_{e}=2200 \mathrm{~K}$ (Figure 8), the gravity has a strong influence on $\mathrm{CN}$; the effect is also noticeable for $\mathrm{CO}$.

In Figure $9\left(T_{e}=3400 \mathrm{~K}\right.$ and $g=0.1,1.0$ and 10$)$, we see that the CN system is not so much influenced by the gravity. On the other hand, the strengths of the bandhead of $\mathrm{CO}$ or of other lines are fairly sensitive to the gravity.

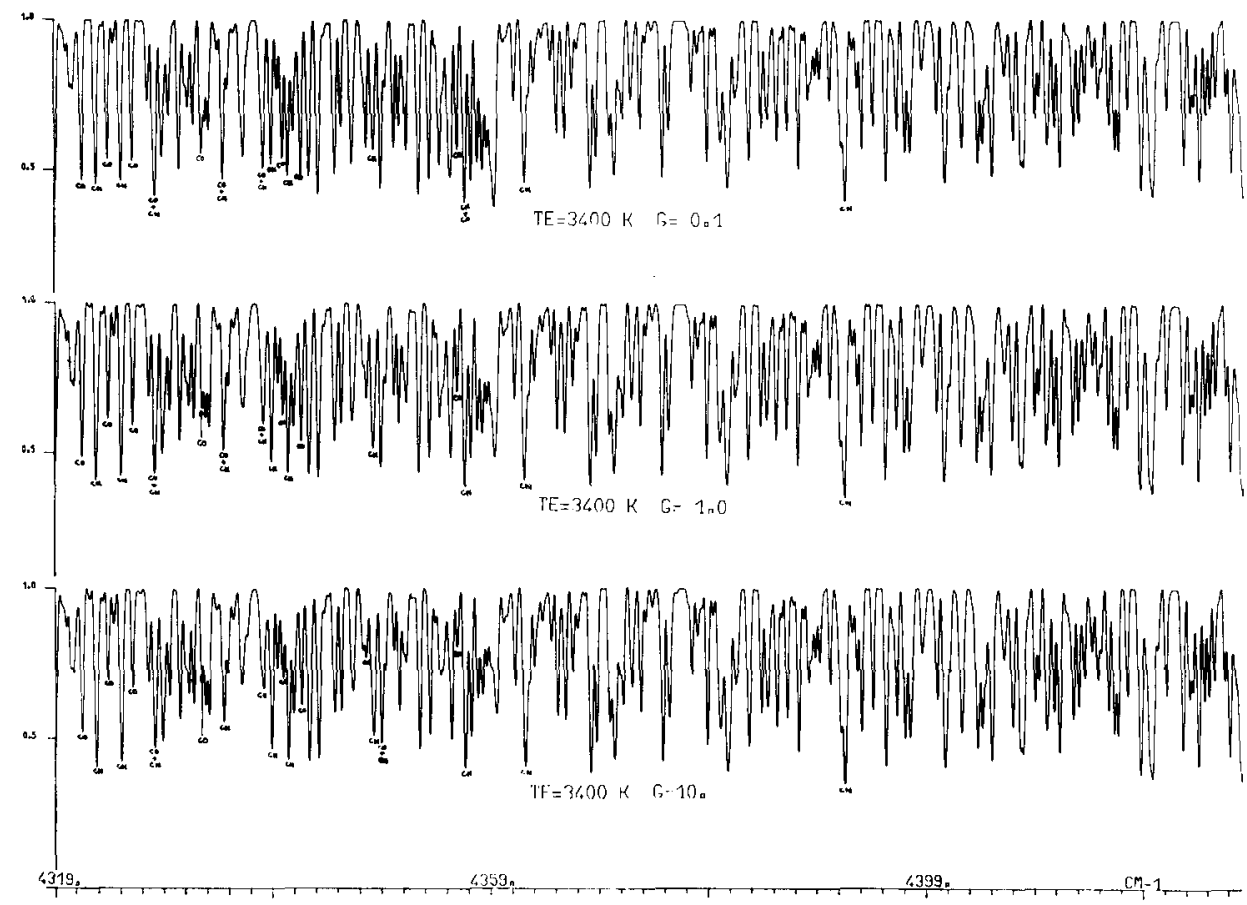

Fig. 9. Influence of the gravity on the synthetic spectra: $g=0.1,1.0$ and 10 for $T_{e}=3400 \mathrm{~K}$. 
For $T_{e}=4200 \mathrm{~K}$ and $g=0.1,1.0$ and 10 (Figure 10), the high effective temperature is not favourable to the formation of molecules. Atomic absorption should be included in the opacity distribution functions. However, we see that the $\mathrm{CO}$ spectrum is stronger for a weak gravity ( $g=0.1$ corresponding to supergiant $\mathrm{C}$ stars), while for $g=10$, the CN spectrum is the stronger.

We may make several remarks:

- All the synthetic spectra have been computed with the same $\mathrm{H} / \mathrm{C} / \mathrm{N} / \mathrm{O}$ abundance ratio. However, the molecular features of $\mathrm{CO}$ and $\mathrm{CN}$ vary strongly and differently with the effective temperature and the gravity. Consequently, we have to be very careful when we determine abundances from slab models of cool stars.

- To explain the behaviour of the two molecular systems, it is necessary first to compare the synthetic spectra with the $T(\tau)$ laws and the run of the partial pressure through the atmospheres, in order to see the variation of the abundances of the two molecules with depth in the atmosphere; secondly, it is necessary to study how these molecular bands evolve with the temperature.

- Some synthetic spectra computed over correctly chosen spectral ranges, are good tools for the determination of effective temperatures and gravities.

It must be pointed out that when studying line blanketing in cool atmospheric layers, the effect of the cut off for weak lines has to be considered very carefully before making comparisons between computed and observed stellar spectra. It is

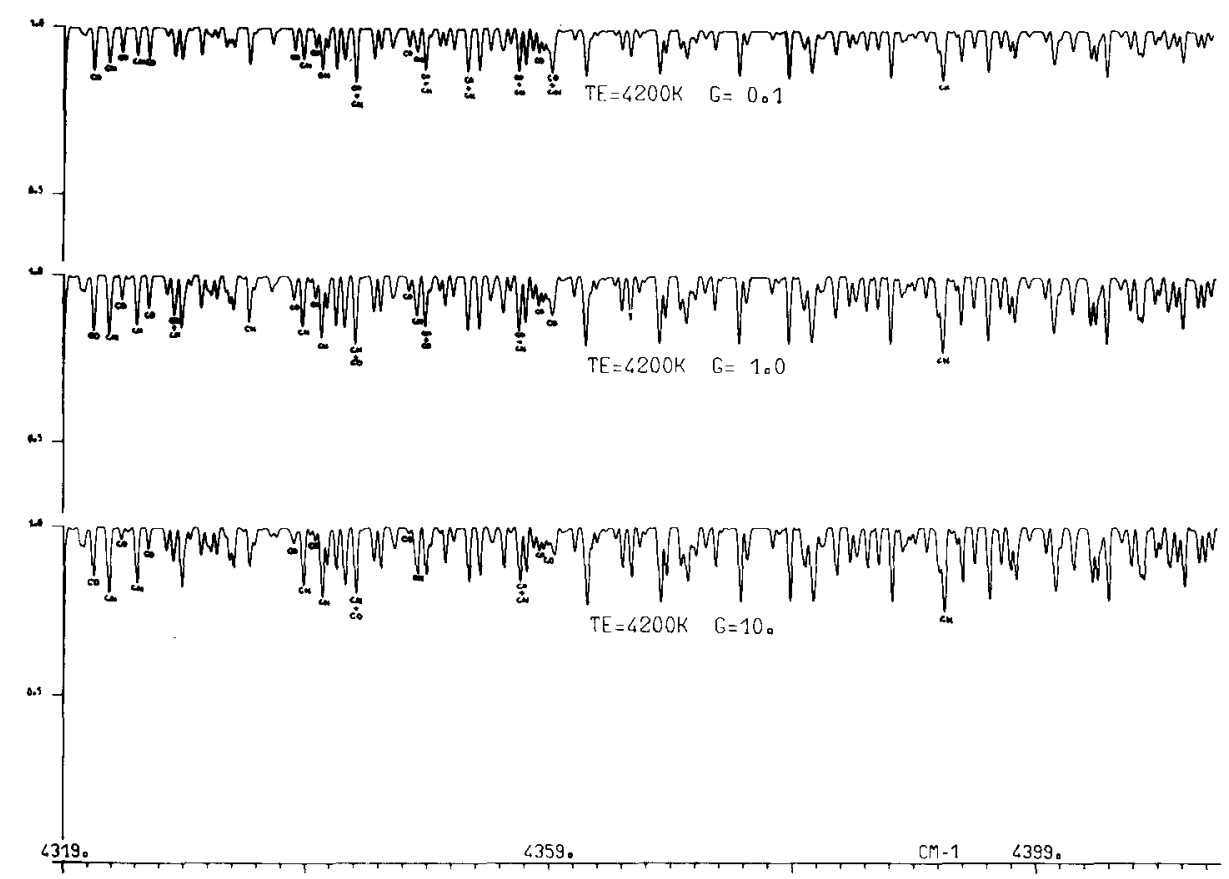

Fig. 10. Influence of the gravity on the synthetic spectra: $g=0.1,1.0$ and 10 for $T_{e}=4200 \mathrm{~K}$. 


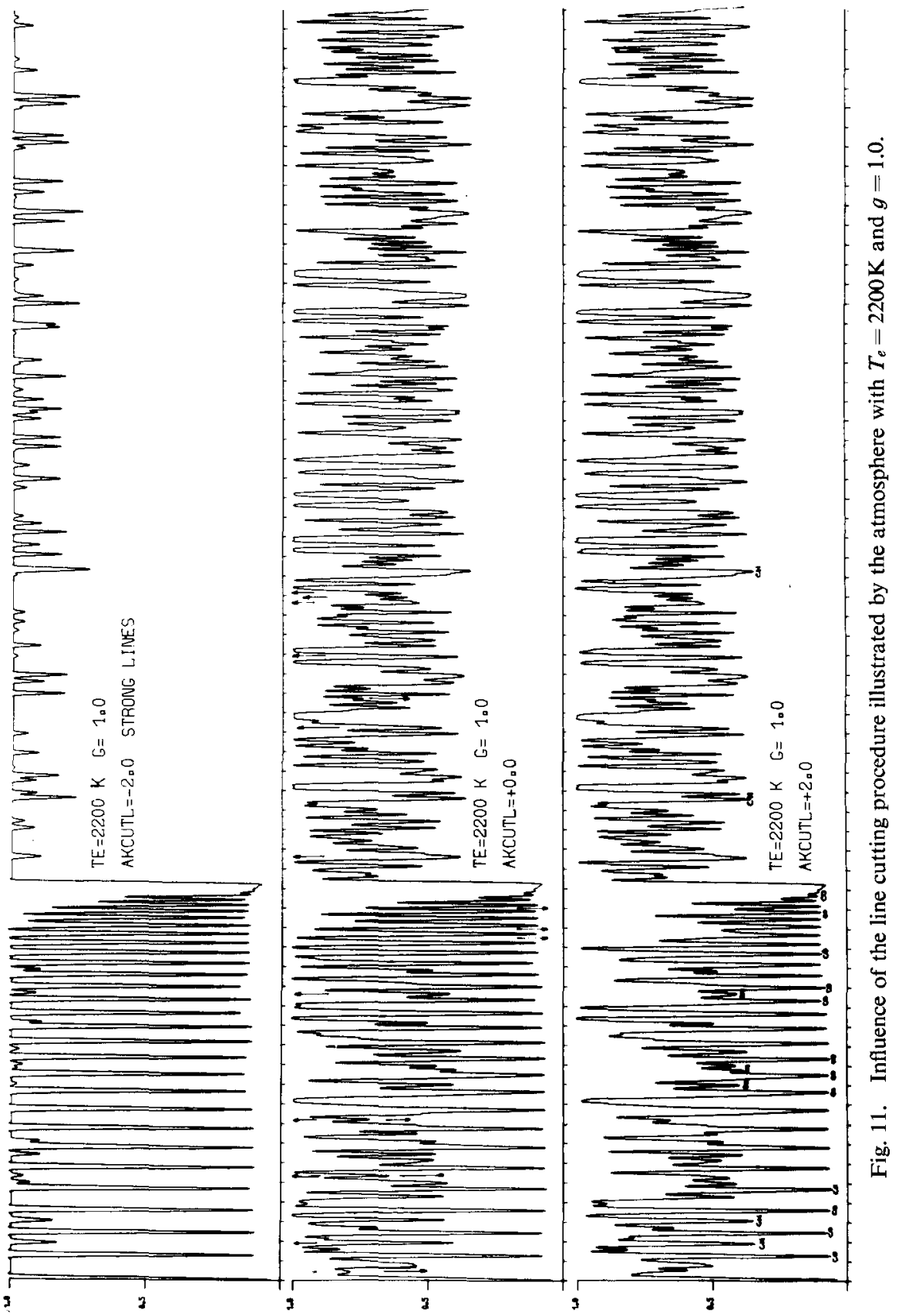




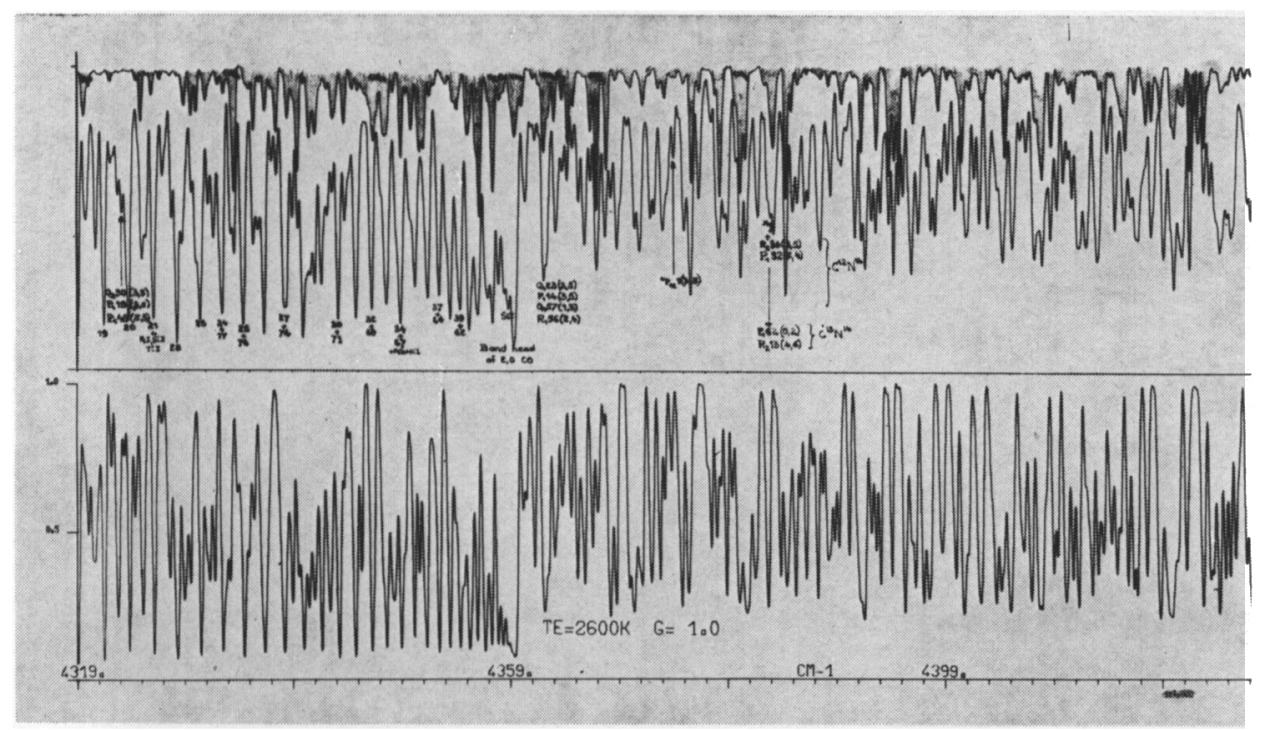

Fig. 12. At the top, solar spectrum; in the middle, spectrum of the carbon star UU Aur; at the bottom, synthetic spectrum computed with $T_{e}=2600 \mathrm{~K}$ and $g=1$.

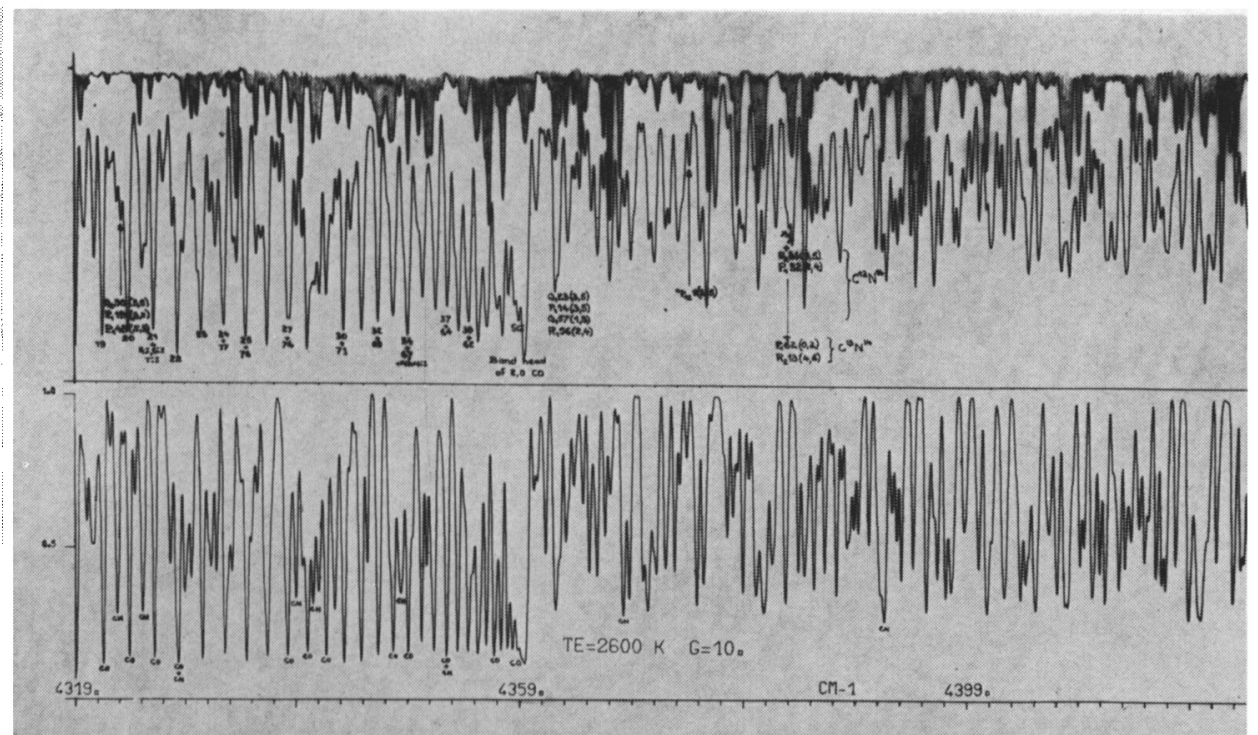

Fig. 13. Idem quod Figure 12, except that the synthetic spectrum has been computed with $T_{e}=2600 \mathrm{~K}$ and $g=10$. 


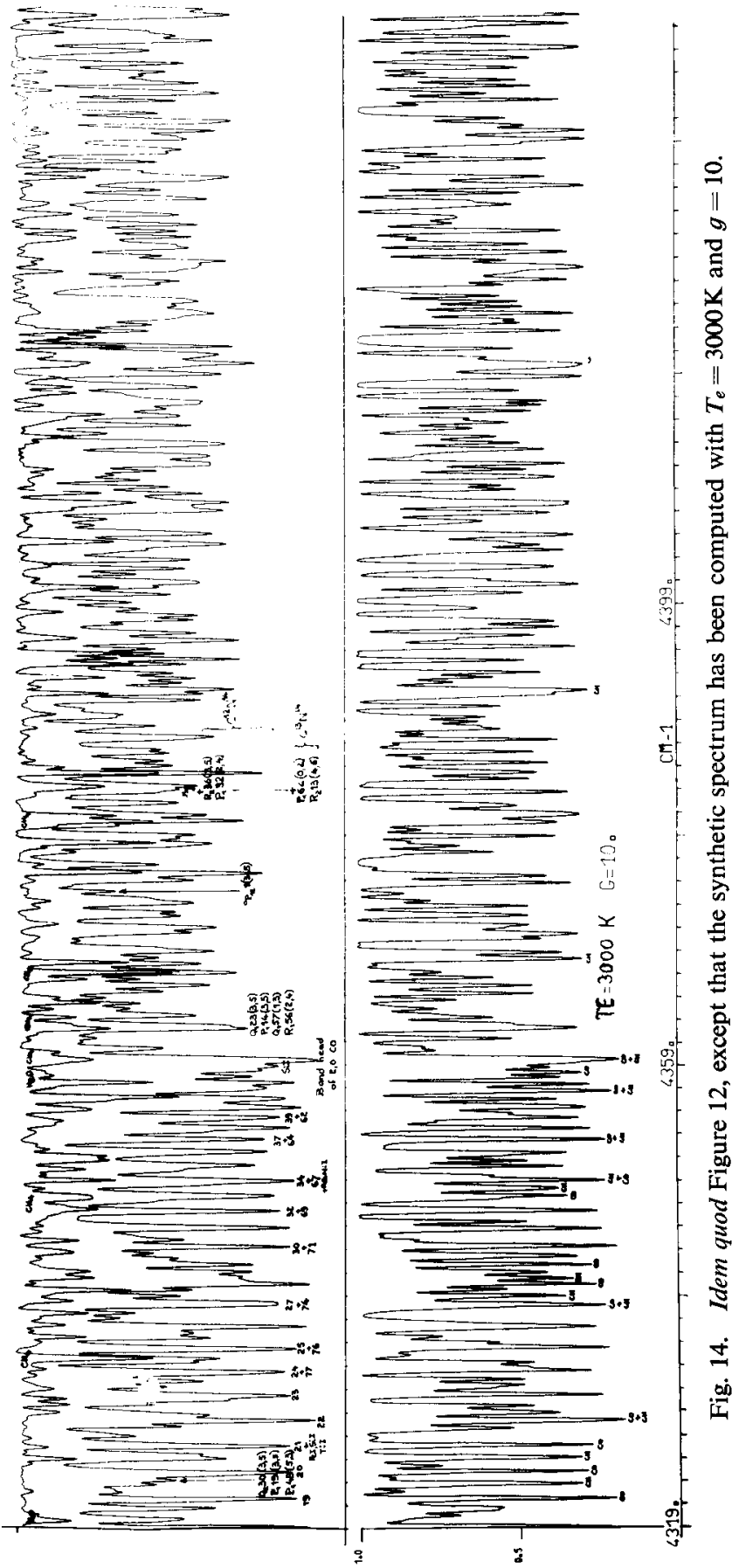


useful to show the effects of the line cutting procedure $\left(l_{v}<k_{v} \times 10^{\mathrm{AKCUTL}}\right)$ for the weak lines and for the wings of strong lines.

Figure 11 has the following characteristics: $T_{e}=2200 \mathrm{~K}, g=1.0$ and AKCUTL= $=-2,0,+2$. For this temperature, the cutting procedure has little influence on the strong lines but a strong influence on the weak lines, when AKCUTL is positive. This is a further reason for thinking it necessary to include, in the coolest model atmospheres, the weak lines furnished by polyatomic molecules. It is evident that the appearance of the spectrum varies with the cut adopted.

In the next three figures, we compare the spectrum of the carbon star UU Aur, obtained at a resolution of $0.3 \mathrm{~cm}^{-1}$ by Maillard in 1972, with some synthetic spectra. In these figures, we show, at the top, the solar spectrum, the lines of which blend the stellar spectrum, then the stellar spectrum itself, and finally the synthetic spectrum for $T_{e}=2600 \mathrm{~K}$ and $g=1$ (Figure 12), for $T_{e}=2600 \mathrm{~K}$ and $g=10$ (Figure 13) and for $T_{e}=3000 \mathrm{~K}$ and $g=10$ (Figure 14).

From the comparison of the most prominent features of the observed and synthetic spectra, we suggest that UU Aur has $T_{e}=2600 \mathrm{~K}$ and $g=10$. Baumert (1972) obtains a similar value by narrow band photometry. We may refine these values of the effective temperature and the gravity by studying bands of less strong molecular system that could be more sensitive to these parameters, such as the $C_{2}$ Ballik-Ramsay system.

\section{Conclusion}

We note that for the high temperature model atmospheres, we should include the visible and the infrared atomic lines, and for the cooler ones we should include polyatomic molecules and grains. Departures from LTE ought to be examined, as was shown by Querci (1972) and emphasized by Thompson (1973).

Attempts in these directions are being made at Meudon. The identification of the infrared atomic lines on high resolution cool star spectra is being currently pursued (see, for example, Chauville et al., 1970). We have recently identified some titanium lines arising from two electron jumps, the interpretation of which is not going to be easy. The computation of atomic oscillator strengths are made following Decker's formulation (1969). Many more or less sophisticated methods are currently available for the computation of the atomic radial wavefunctions.

As to the molecules, everyone working in this field realises that we lack a great deal of data on wavelengths and oscillator strengths for the molecules of astrophysical interest, especially in the infrared region. In our laboratory, we are studying the $\mathrm{C}_{2}$ Ballik-Ramsay spectrum by Fourier Transform Spectroscopy with a high resolution interferometer devoted to laboratory spectra. We plan to extend this work to other carbon and oxygen molecules, and similarly to atomic spectra.

\section{Acknowledgements}

We would like to thank DrT. Tsuji for his contribution to the construction of model 
atmospheres and for many helpful discussions. Mrs F. Gautier from CIRCE (Orsay) is greatly thanked for her help in the drawing of the spectra.

\section{References}

Auer, L.: 1967, Astrophys. J. Letters 150, L53.

Baumert, J. H.: 1972, Ph.D. Thesis, The Ohio State University.

Chauville, J., Querci, F., Connes, J., and Connes, P.: 1970, Astron. Astrophys. Suppl. 2, 181.

Decker, E.: 1969, Astron. Astrophys. 1, 72.

Querci, F.: 1972, Thèse d'Etat, Université de Paris.

Querci, F., Querci, M., and Tsuji, T.: 1974, Astron. Astrophys., in press.

Querci, M., Querci, F., and Tsuji, T.: 1972, Mem. Soc. Roy. Sci. Liege, 6th Ser. 3, 179.

Ström, S. E. and Kurucz, R. L.: 1966, J. Quant. Spectrosc. Radiat. Transfer 6, 591.

Thompson, R. I.: 1973, Astrophys. J. 181, 1039.

Tsuji, T.: 1973, Astron. Astrophys. 23, 411.

\section{DISCUSSION}

Hyland: I am very interested in the model atmospheres and emergent fluxes that you derive, in particular from the point of view that the temperature scale of carbon stars is just not known, and it is a very important one, and therefore the difference is that your model atmospheres compared with Alexander and Johnsons - it is very important to clear up why there is such a difference. Do you have any ideas on this?

Querci: For me narrow band photometry is good, but I prefer to see a problem with high resolution spectra. We try to pick up some better spectral intervals. I have one computed curve in terms of UBV and so on, and sometimes between two spectral intervals. You can see this in the diagram.

Hyland: A lot of us use further out, like JHKL bands, and the fact that yours and Johnson's differ does not depend on our observations. We would like to know which ones are right, and why.

Querci: But I think this is not a good model for making comparisons. When the temperature decreases all the bands of the visual are very important. 